\title{
Semantic WebBased Analysis of Product Line Variant Model
}

\author{
Shamim Ripon, Senior Member, IACSIT, M. M. Piash, S. M. A. Hossain, and M. S. Uddin
}

\begin{abstract}
Feature diagrams are widely used to model software product line (SPL) variants. However, there is a lack of precisely defined formal notations for representing and verifying such models. Several proposals have been made in recent years to model product line features. In our earlier work we have presented a product line model to model and customize products from product specific features facilitating the very concept of reuse of common features throughout product family. However, no formal verification has been proposed for such product line model. This paper presents an approach to modeling and analyzing SPL model using semantic-web approach. We use OWL-DL to model the common and variant features in the SPL model. A reasoning tool is then used to verify the consistency of the feature configuration in the model. Such formal checking confirms and strengthens the variability model that has been presented in our earlier work. Besides, the OWL-DL representation also facilitates the search and maintenance of feature models and support knowledge sharing within a reusable engineering context.
\end{abstract}

Index Terms-Variability model, semantic web, product line, feature diagram, OWL, OWL-DL, racer.

\section{INTRODUCTION}

Designing, developing and maintaining a good software system is a challenging task still in this 21 st century. The approach of reusing existing good solutions for developing any new application is now one of the central focuses of software engineers. Building software systems from previously developed components saves cost and time of redundant work, and improves the system and its maintainability. A new software development paradigm, software product line [1], is emerging to produce multiple systems by reusing the common assets across the systems in the product line. However, the idea of product line is not new. In 1976 Parnas [2] proposed modularization criteria and information hiding for handling product line.

Core assets are the basis for software product line. The core assets often include the architecture, reusable software components, domain models, requirements statements, documentation and specifications, performance model, etc. Different product line members may differ in functional and non-functional requirements, design decisions, run-time architecture and interoperability (component structure, component invocation, synchronization, and data communication), platform, etc. The product line approach integrates two basic processes: the abstraction of the commonalities and variabilities of the products

Manuscript received April 9, 2013; revised July 9, 2013.

The authors are with the Department of Computer Science and Engineering, East West University, Dhaka, Bangladesh (e-mail: dshr@ ewubd.edu) considered(development for reuse) and the derivation of product variants from these abstractions (development with reuse) [3].

Common requirements among all family members are easy to handle and can be integrated into the family architecture and are part of every family member. But problem arises from the variant requirements among family members. Variants are usually modeled using feature diagram, inheritance, templates and other techniques. In comparison to analysis of a single system, modeling variants adds an extra level of complexity to the domain analysis. Different variants might have dependencies on each other. Tracing multiple occurrences of any variant and understanding their mutual dependencies are major challenges during domain modeling. While each step in modeling variants may be simple but problem arises when the volume of information grows. As a result, the impact of variant becomes ineffective on domain model. Therefore, product customization from the product line model becomes unclear and it undermines the very purpose of domain model.

In our earlier work [4], we have presented a variability model to draw the common and variant features of a product line. In the variability model, a tabular based approach [5] has been used in conjunction with feature model to portray the reusable features of product line. The tabular mechanism also provides a decision table supporting product customization. However, the variability model lacks logic based formal definition. A formal verification of such variability model ensures a sound and consistent variability model facilitating a verified product customization mechanism.

To capture domain knowledge and common vocabularies in any field ontologies have shown itself an acceptable paradigm [6]. It is also necessary to process and exploit knowledge in a computer system. Among the various available approaches for knowledge representation, ontologies is a promising solution due to its ability to make the domain knowledge computer readable and processable. Besides, various inference algorithms and tools are available to infer new knowledge from the existing.

Semantic web technology provides a meaningful and shared ontological description of the domain. Web Ontology Language (OWL) [7] is one of the most expressive languages for specifying, publishing and sharing ontologies. OWL not only facilitates better machine interoperability than that of XML, RDF, RDFS etc. but also has formal semantics and support for defining additional vocabulary. Among the various available dialects, this paper uses OWL-DL which is based on Description Logic (DL) [8]. Description logic has already been successfully applied to solve various complex configuration problems [8], [9] as well as to check consistency in UML diagrams [10]. 
This paper formally models and verifies our previously defined variability mode of SPL using semantic web mechanism, OWL-DL in particular. Semantic web mechanism integrates meaningful description and semantic information into SPL models. We use Protégé [11] graphical interface for ontology editing and visually displaying both feature models and feature properties. RACER [12] tool is used to check the consistency of the OWL-DL ontology definitions and feature configurations. We illustrate the modeling by using a case study of "Hall Booking System" product family [4].

The rest of the paper is organized as follows. Section II gives a brief overview of the Hall Booking System. Section III describes the product line model and its table based variant and decision model. We then illustrate the steps of how the common and variant features in the variability model are modeled by using OWL-DL in Section IV. Section V shows the automated analysis of OWL-DL representation of feature configurations. After outlining a brief review of related works in Section VI, we conclude the paper and outline our future plans in Section VII.

\section{HALl BOOKING SYSTEM OVERVIEW}

We use Hall Booking System family to illustrate our variability modeling mechanism. The system is used in academic institutions to reserve tutorial rooms and lecture halls, at companies to reserve meeting rooms, and at hotels to reserve rooms and conference facilities, etc. In another sense, the system can be used for either academic or non-academic purposes. Users can manage their own reservation with the system. The main purpose and the core functionality are similar across the Hall Booking System family; however, there are many variants on the basic theme. One of the basic variants is the charging of booking system. Whenever the system is used for academic purposes, no charge is needed for booking halls, whereas there may be a need to charge for booking halls in other areas. In some systems, there are facilities available for seasonal booking as well as multiple bookings. Our Hall Booking System default models include the following functional features:Make reservation, Modify reservation, Search/Retrieve reservation, Add a resource (Hall), Delete a resource (Hall), Modify a resource, Search/receive a Hall.

By using the extensions shown in [13], a part of the features of Hall Booking System is shown in Fig. 1. Mandatory features appear in all the members of the family whereas variant features appear in some members of the family. Variant features are also classified as Optional, Alternative and Or features. An optional feature may or may not be part of a system. An example of optional feature is Reservation Charge. An alternative feature describes the selection of one from many features. An example of alternative feature is Reservation Mode which can be either Single or Block. An or-feature describes any of many features. For example a Block Reservation can be made by multiple rooms or multiple times or by both. Variants may depend on other variants. Two types of dependencies are illustrated in this paper: requires and exclude.

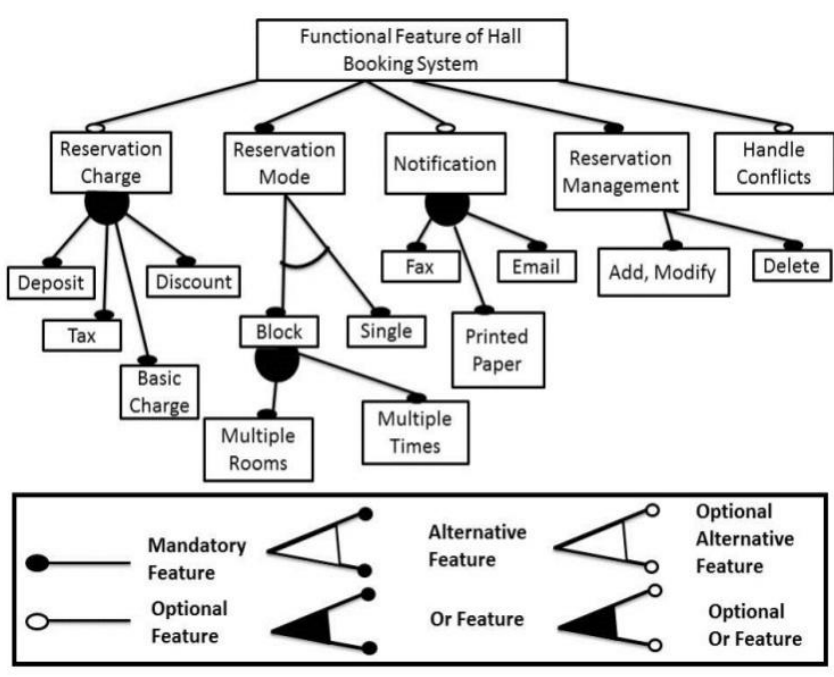

Fig. 1. Hall booking system feature diagram

\section{VARIABILITY MODEL}

In developing product line, the variants are to be managedin domain engineering phase, which scopes the product lineand develops the means to rapidly produce the members ofthe family. It serves two distinct but related purposes, firstly, it can record decisions about the product as a whole includingidentifying the variants for each member and secondly, it cansupport application engineering by providing proper informationand mechanism for the required variants during productgeneration.

In our approach, we initially consider a domain modelwhich includes default domain view, a variant model andcustomization requirements. Default domain views describetypical system in a domain. Default domain views are thestarting point for understanding the scope of the product line,i.e., the range of systems in the domain we wish to consider.We draw a model to represent the variants of a product line.The model contains all the variant related information requiredfor customizing any product. After getting the requirementsfor any particular product of the product line, the productline model collects proper variant information from the variantmodel. A flexible variant configuration tool (FVC) interpretsthe variant model and customizes the default domain modelby adapting and customizing the default domain according tothe particular product requirements. Fig. 2 gives a top levelview of the targeted variant model along with its position andactivity with product line model.

The left-hand-side of Fig. 2 depicts the product line model which comprises the default model and the variants. A feature diagram can be drawn from the product line model to get an overall picture of the product line functionalities, both common and variants. The right-hand-side of the figure mainly depicts the variant model. The variant model is constructed by getting information from the product line relating to both common and variant features. A generic domain model is created by adding the variants with the default model and during its construction information is collected from the variant model and also from the product line model. Finally, the required product model is developed by customizing the generic domain model after handling the variants according to the product requirements. 


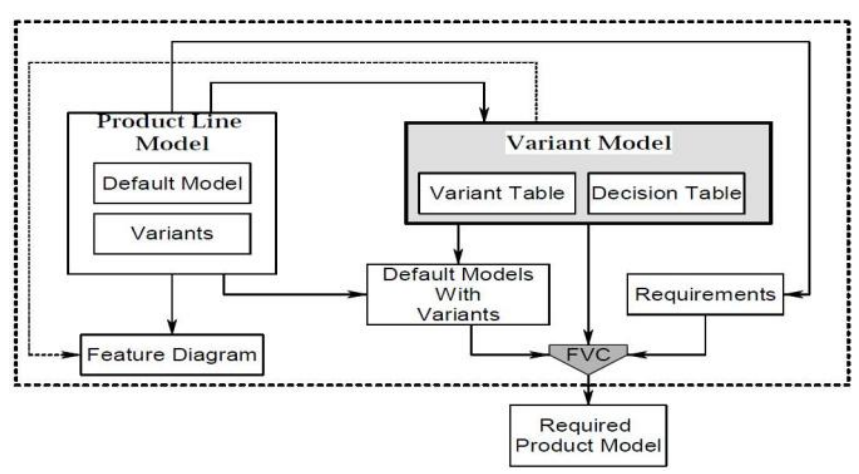

Fig. 2. Variant model within product line model

We represent the variant model by using a unified tabularapproach. The table contains all the variant related informationalong with the commonalities of the domain. A decision tableis constructed based on the available variant information. Thedecision table is used to derive customized product fromthe product line model. Feature diagram, on the other hand,represents the common and variant requirements in a tree likestructure. However, detailed information regarding variantsand their dependencies cannot be represented as much astabular method. Tabular method can be augmented with featurediagram. As the table and the associated decision table do nothave any logical (or formal) definition any subsequent formalverification and automated consistency checking of the variantmodel cannot be performed. After modeling the variant tableand decision table we provide logical definition of the featurediagram and perform various analysis operations.

The table is constructed by keeping all the information available in FODA feature diagram. By analyzing the characteristics of variants of a domain we found that all the variants follow certain types of common categorization and all of them have certain behavior. So, if the variants are arranged according to these categorizations and behavior then they can be handled easily in a domain. After identifying the common characteristics and behavior of variants we arranged the variants in a tabular format. The table contains the following information.

- Variant - the name of each variant

- Variant type - the type of each variant (Mandatory or Optional)

- Sub domain - the applicable area of the variant and its values

- Relation among values - the relationship among the values of each variant (Alternative, OR)

- Dependency -the variant or variant values, on which the variant is dependent.

Usually, there are some complex dependencies among variants, and feature diagrams cannot represent them properly. We used the notations given in [7]. A partial variant table ofHall Booking system is depicted in Fig. 3.

A decision table is then derived from the variant model. A small part of the decision table is given in Fig. 4. In the table, each variant is placed in a column, its description, possible choices and traces to the variant are shown in other columns. When inclusion or exclusion of any variant depends on a variant or its values then that variant is placed in the subordinate position showing the values for which it can be chosen. For example, Reservation Mode variant has two possible values either Single or Block. So these two valuesare placed in the subordinate position of the parent variant(Reservation Mode) in the decision table. Similarly whenBlock reservation is to be considered then it needs to check itsvalues (Multiple Room and Multiple Time) and these valuesalong with their other information will be also be subordinated.For each variant and for each of their values, the decision tablecan have a corresponding entity.

\begin{tabular}{|c|c|c|c|c|}
\hline Variant & Values of variant & Relations & Applicable Area & Dependency \\
\hline $\begin{array}{l}\text { V1. } \\
\text { Reservation } \\
\text { Mode }\end{array}$ & $\begin{array}{l}\text { V1.1 Single } \\
\text { V1.2 Block }\end{array}$ & Alternative & All & None \\
\hline $\begin{array}{l}\text { V2. } \\
\text { Reservation } \\
\text { Charge }\end{array}$ & $\begin{array}{l}\text { V2.1 Deposit } \\
\text { V2.2 Tax } \\
\text { V2.3 Discount } \\
\text { V2.4 Refund }\end{array}$ & OR & Non Academic & None \\
\hline $\begin{array}{l}\text { V3. } \\
\text { Block } \\
\text { Reservation }\end{array}$ & $\begin{array}{l}\text { V3.1 Multiple Room } \\
\text { V3.2 Multiple time }\end{array}$ & OR & All & V1.2 \\
\hline $\begin{array}{l}\text { V4. } \\
\text { Notification }\end{array}$ & $\begin{array}{l}\text { V4.1 Fax } \\
\text { V4.2 Email } \\
\text { V4.3 Printed Paper }\end{array}$ & OR & All & None \\
\hline $\begin{array}{l}\text { V5. } \\
\text { Reservation } \\
\text { Discount }\end{array}$ & $\begin{array}{l}\text { V5.1 Block Discount } \\
\text { V5.2 Seasonal } \\
\text { discount } \\
\end{array}$ & OR & Non Academic & $\mathrm{V} 2.3, \mathrm{~V} 1.2$ \\
\hline
\end{tabular}

Fig. 3. Part of hall booking system variant table

\begin{tabular}{|l|l|l|l|c|}
\hline \multicolumn{1}{|c|}{ Variant } & \multicolumn{2}{|c|}{ Description } & \multicolumn{1}{c|}{ Values } & Traces \\
\hline $\begin{array}{l}\text { Reservation } \\
\text { Mode }\end{array}$ & \multicolumn{2}{|c|}{ What is the reservation mode? } & Single, Block & V1 \\
\hline $\begin{array}{l}\text { Block } \\
\text { reservation }\end{array}$ & Block & $\begin{array}{l}\text { What is the type of } \\
\text { block reservation? }\end{array}$ & $\begin{array}{l}\text { Multiple Room, Multiple } \\
\text { Time }\end{array}$ & V3 \\
\hline $\begin{array}{l}\text { Reservation } \\
\text { charge }\end{array}$ & Single & $\begin{array}{l}\text { How is the charge for } \\
\text { reservation? }\end{array}$ & Deposit, Tax, Discount & V2 \\
\hline
\end{tabular}

Fig. 4. Part of hall booking system variant table

So, after getting requirements from stakeholders, those are checked with the variant model and the decision table. The variability model will guide the application engineer to properly choose the required variants very easily. Therefore, the overall product generation process will be faster and less erroneous.

\section{Modeling Variant Model Using OWL-DL}

We have presented system family model using tabular method in earlier section. As it is not convenient to express all variant related information, a feature diagram can beaugmented with a table based variant model. Both feature diagram and tabular method can complement each other as well as both can be used alternatively. We present semantic web based ontological definitions of the feature diagram to give a formal definition to the variant model and decision table.

There are six types of feature relations available in a feature diagram. By using OWL-DL we model six types of relations, namely mandatory, optional, alternative, or, optional alternative and optional or. Two additional constraints: requires and excludes are also modeled.

First, OWL ontology is built for the nodes and edges in the feature diagram. After identifying the nodes of concepts and features in the feature tree, each node is modeled as an OWL class. Each class is defined as mutually disjoint. For each of these nodes we create a Rule class. There are two kinds of conditions in Rule class, firstly, a necessary and sufficient condition and secondly, a number of necessary constraints indicating various relationship between parent and child. In the final step of ontology definitions, an object property is created for each type of edges in the feature diagram. The 
TABLE I: SUMMARY OF OWL SYNTAX

\begin{tabular}{cl}
\hline Notation & \multicolumn{1}{c}{ Explanation } \\
\hline $\mathrm{T}$ & Superclass of all OWL classes \\
$A \subseteq B$ & $A$ is a subclass of $B$ \\
$A \sqcap B$ & $A$ and $B$ are disjoint \\
$A \sqcup B$ & Class intersection \\
$A \equiv B$ & Class union \\
$\mathrm{T} \sqsubseteq \forall P \cdot A$ & Class equivalence \\
$\mathrm{T} \sqsubseteq \exists P \cdot A$ & Range of property is a class $A$ \\
& allValuesFrom/someValuesFrom restriction, giving the \\
& class that for every instance of this class that has \\
& instances of property P, all/some of the values of the \\
& property are members of the class A
\end{tabular}

OWL syntax used in this paper is summarized in Table I. For a parent feature $A$ and for each of its child features $B_{1}, B_{2}, \ldots, B_{n}$ the initial modeling produces the following ontology,

$$
\begin{array}{ll}
A \sqsubseteq \mathrm{T} & \text { hasA } \subseteq \text { ObjectProperty } \\
\text { ARule } \sqsubseteq \mathrm{T} & \mathrm{T} \sqsubseteq \forall \text { has } A \cdot A \\
& \text { ARule } \equiv \exists \text { has } A \cdot A \\
B_{i} \sqsubseteq \mathrm{T} & \text { has } B_{i} \sqsubseteq \text { ObjectProperty } \\
B_{i} \text { Rule } \sqsubseteq \mathrm{T} & \mathrm{T} \sqsubseteq \text { has } B_{i} \cdot B_{i} \\
& B_{i} \text { Rule } \equiv \text { has } B_{i} \cdot B_{i} \\
A \subseteq B i \text { for } 1 \leq i \leq n & \\
B i \sqsubseteq \neg B j, \text { for } 1 \leq i, j \leq n \wedge i \neq j
\end{array}
$$

\begin{tabular}{|c|c|}
\hline Feature Types & OWL-DL Representation \\
\hline Mandatory & ARule $\subseteq \exists$ has $B_{i} \cdot B_{i} \quad$ for $l \leq i \leq n$ \\
\hline B & 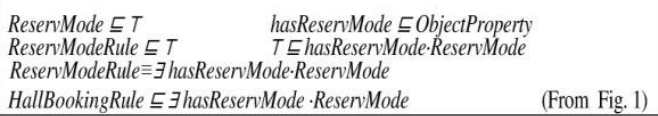 \\
\hline Optional & $B_{i} \subseteq T \quad$ has $B_{i} \subseteq$ ObjectProperty \\
\hline & $B_{i}$ Rule $\subseteq T \quad B_{i}$ Rule $\equiv \exists$ has $B_{i} B_{i} \quad$ for $1 \leq i \leq n$ \\
\hline$B$ & $\begin{array}{llr}\text { ReservCharge } \sqsubseteq T & \text { hasReservCharge } \sqsubseteq \text { ObjectProperty } & \text { (From Fig. 1) } \\
\text { ReservChargeRule } \sqsubseteq T & \text { ReservChargeRule } \equiv \text { HhasReservCharge } \cdot \text { ReservCharge }\end{array}$ \\
\hline Alte & 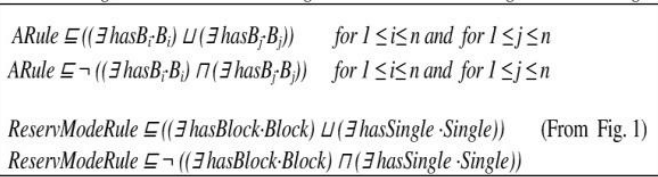 \\
\hline & $\begin{array}{l}\text { ARule } \sqsubseteq U\left(\exists \text { has } B_{i} \cdot B_{i}\right) \text { for } 1 \leq i \leq n \\
\text { ReservChargeRule } \sqsubseteq((\exists \text { hasDeposit.Deposit }) \sqcup(\exists \text { hasTax } \cdot \text { Tax }) U \\
(\exists \text { hasBasic_Charge } \cdot \text { Basic_Charge }) U(\exists \text { hasDiscount } \cdot \text { Discount })) \text { (From Fig. } 1)\end{array}$ \\
\hline $\begin{array}{l}\text { Optional } \\
\text { Alternative }\end{array}$ & 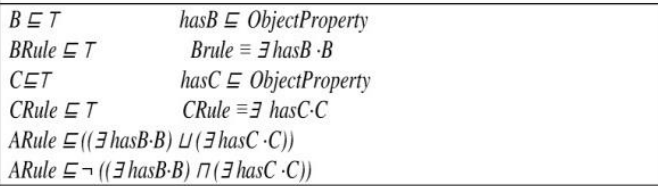 \\
\hline Optional Or & 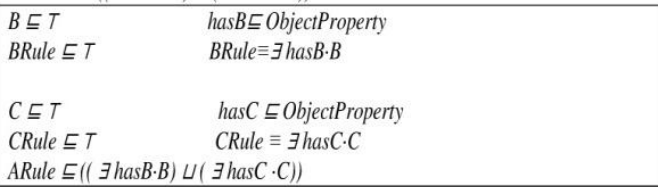 \\
\hline $\begin{array}{l}\quad \text { Requires } \\
\text { In Fig. 1, if 'Fax' } \\
\text { requires 'Printed } \\
\text { Paper'. }\end{array}$ & $\begin{array}{l}\text { ARule } \subseteq \exists \text { has } B_{i} B_{i} \quad \text { for } l \leq i \leq n \\
\text { FaxRule } \subseteq T \\
\text { FaxRule } \equiv \exists \text { hasFax.Fax } \\
\text { Fax } \subseteq \text { JhasPrinted_Paper.Printed_Paper }\end{array}$ \\
\hline Excludes & ARule $\subseteq \neg\left(\exists\right.$ has $\left.B_{i} B_{i}\right)$ \\
\hline
\end{tabular}

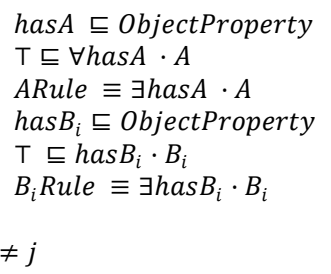

Now we are ready to model the feature relations using the ontology. Table II shows the OWL-DL representation of six types of features and two constraints of a feature model.

TABLE II: OWL-DL REPRESENTATION OF VARIOUS TYPES OF FEATURES

\section{Feature Configuration Verification}

We input our ontology into Protégé and use RACER [12] to check its consistency. For the initially encoded ontology, RACER checks for consistency and show that encoded definitions are consistent.

In feature modeling, an instance of a concept is a configuration derived from the feature model. In order to detect inconsistency in a configuration OWL classes are used, and features and concept instances are then simulated. When an instance is checked, the reasoner not only can check inconsistency but also shows which class/classes are inconsistent. We use an existential restriction for each feature included in the configuration. For each feature $B$ available in a feature diagram but not in its configuration, we use a $\neg \exists$ has $B . B$ restriction to prevent the reasoning engine from inferring the existence of this feature in the configuration.

If an instance $C$ of a concept derived from a feature diagram with root concept $A$ and a set of features $B 1, B 2, \ldots, B i$ assuming that they appear in the configuration of $X$ and the features $B i+1, \ldots, B n$ do not, the feature configuration can be modeled as follows,

$$
\begin{gathered}
X \sqsubseteq \text { ARule } \\
\left.X \equiv \Pi\left(\exists \text { hasB }_{j} \cdot B_{j}, \text { for } 1 \leq j \leq i\right)\right) \sqcap \\
\Pi\left(\neg \exists \text { has } B_{k} \cdot B_{k}, \text { for } i<k \leq n\right)
\end{gathered}
$$

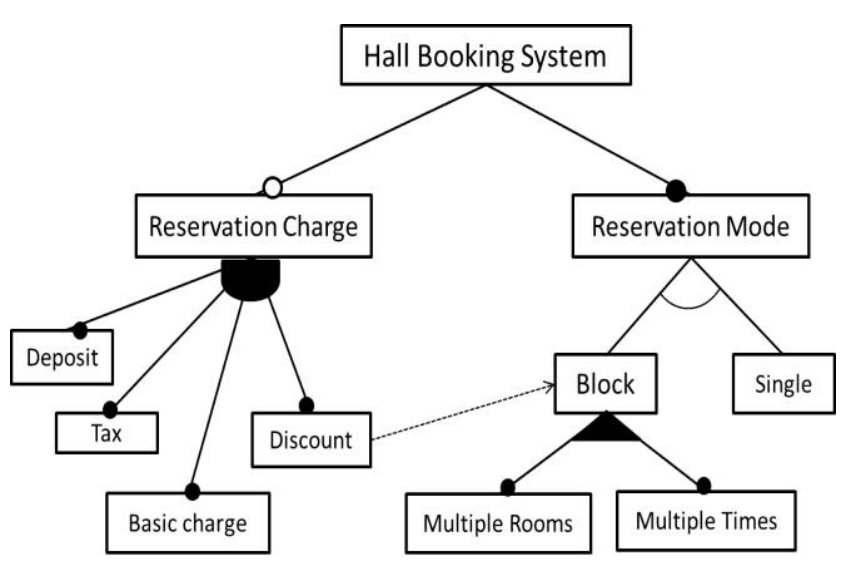

Fig. 1. Partial feature tree with 'Requires' relation

Let us consider a 'requires' relation between the features 'Discount' and 'Block' from Reservation Mode. A part of the figure is shown in Fig. 5 where the 'requires' relation is shown by using a dotted line from 'Discount' to 'Block'.

\section{DiscountRule $\subseteq T$ \\ DiscountRule $\equiv \exists$ hasDiscount $\cdot$ Discount \\ DiscountRule $\sqsubseteq \exists$ hasBlock $\cdot$ Block}

Suppose the configuration containing a concept instance $E$ and some features for the feature diagram in Fig. 5. We call the instance node the class $E$. The feature 'Block' requires 'Discount', but in the instances if 'Block' is not selected the reasoned tool shows the configuration is inconsistent.

$$
\begin{gathered}
E \equiv \text { HallBooking } \\
E \equiv((\exists \text { hasReservCharge } \cdot \text { ReservCharge }) \\
\Pi(\exists \text { hasDiscount } \cdot \text { Discount }) \\
\Pi(\exists \text { hasReservMode } \cdot \text { ReservMode }) \\
\Pi(\neg \text { hasBlock } \cdot \text { Block }))
\end{gathered}
$$




\section{RELATED WORK}

Formal representation and verification of feature model is a challenging task. Various formal approaches have been adopted over the years for this purpose. Logic based reasoning has played a major role so far for checking various analysis operation [14], [15]. Propositional and First-Order Logic (FOL) are being used in [16]-[20] to specify the features and their relationships and constraints. Automated tools [18], [21] based on such logic are also been developed to automatically check feature consistency and valid configuration of feature in SPL.

While most approaches applied FOL, very few used semantic web and ontology to model and analyze feature model. Semantic web not only provide semantic foundation but also facilitates model creation, verification, integration and maintenance. Similar to our approach OWL-DL is also being used in $[22,23]$ to analyze feature diagram. Along with modeling feature using OWL-DL both of them proposed additional tool support to design feature models. Comparing to these approaches we not only use OWL-DL not check feature consistency, but also plan to check both domain and product level feature configurations to check the analysis operations suggested in [15].

\section{CONCLUSION}

Successful development of software system families requires appropriate organization and management of the products involved. A significant characteristic of developing system families is the management of common and variant features, a crucial success factor of system family approach. We presented a verification approach of product line model by using semantic web technology. OWL-DL is used to represent feature models and configuration in a concise and unambiguous way. Features are represented as OWL classes and relations as properties.

OWL ontologies provide a suitable platform for the development of semantically aware software product line allowing the knowledge within the feature model to be shared among the reusable features of the SPL. We presented our preliminary result of consistency checking using RACER. A through consistency checking is currently undergoing. The OWL-DL verification of the feature models of system family gives confidence of our previously defined variability model.

We have focused on the modeling and verification of only functional features of SPL. Non-functional requirements play a crucial role in successful software development. Our future plans include the incorporation of non-functional requirement with functional requirement of a SPL and extend our existing verification strategy to support both functional and non-functional features. We are also interested in web-services based product line management, the orchestration of their feature as well as their feature verification.

\section{REFERENCES}

[1] P. Clements and L. Northrop, Software Product Lines: Practices and Patterns. 3rd ed. Addison-Wesley Professional, August 2001.

[2] D. L. Parnas, Software fundamentals. Boston, MA, USA Addison-Wesley Longman Publishing Co.,Inc., 2001, pp. 193-213.
[3] A. Hein, J. MacGregor, and S. Thiel, "Configuring software product line features," in Proc. the ECOOP 2001 Workshop on Feature Interaction in Composed Systems, Budapest, Hungary, June 18-22, 2001, E. Pulvermuller, A. Speck, J. Coplien, M. D. Hondt, and W. D. Meuter, Ed., vol. 2001-14, 2001, pp. 67-69.

[4] S. H. Ripon, K. H. Talukder, and M. K. I. Molla, "Modeling variability for system families," Malaysian Journal of Computer Science, vol. 16, no. 1, 2003, pp. 37-46.

[5] S. Ripon, "A unified tabular method for modeling variants of software product line," in Pulvermuller, E., Speck, A., Coplien, J., Hondt, M.D., Meuter, W.D., ACM SIGSOFT Software Engineering Notes, Ed. vol. 37, no. 3, 2012, pp. 1-7.

[6] S. Zhang, W. Shen, and H. Ghenniwa, "A review of internet-based product information sharing and visualization," Computers in Industry, vol. 54, no. 1, May 2004, pp. 1-15.

[7] I. Horrocks, P. F. Patel-Schneider, and F. V. Harmelen, "From shiq and rdf to owl: Themaking of a web ontology language," Journal of Web Semantics 1, vol. 1, no. 1, Dec. 2003, pp. 7-26.

[8] F. Baader, D. Calvanese, D. L. McGuinness, D. Nardi, and P. F. Patel-Schneider, The Description Logic Handbook: Theory, Implementation, and Applications, Ed., NY, USA: Cambridge University Press, 2003.

[9] J. S. Dong, C. H. Lee, H. B. Lee, Y. F. Li, and H. Wang, "A combined approach to checking web ontologies," in Proc. the 13th international conference on World Wide Web. WWW '04, NY, USA: ACM, 2004, pp. 714-722.

[10] D. Berardi, "Using dls to reason on uml class diagrams," in Proc. Workshop on Applications of Description Logics, 2002, pp. 1-11.

[11] N. F. Noy, M. Sintek, S. Decker, M. Crubezy, R. W. Fergerson, and M. A. Musen, "Creating semantic web contents with protege-2000," IEEE Intelligent Systems, 2001, pp. 60-71.

[12] V. Haarslev and R. Muller, Racer user's Guide and Reference Manual - Version 1.7.6, 2002.

[13] K. Czarnecki and U. W. Eisenecker, Generative Programming: Methods, Tools, and Applications. NY, USA: ACM Press/Addison-Wesley Publishing Co., 2000.

[14] D. S. Batory, "Feature models, grammars, and propositional formulas," LNCS, J. H. Obbink, K. Pohl, Ed., SPLC., vol. 3714, Springer, 2005, pp. $7-20$.

[15] D. Benavides, A. R. Cortés, P. Trinidad, and S. Segura, "A survey on the automated analyses of feature models," JISBD, 2006, pp. 367-376.

[16] S. Ripon, K. Azad, S. J. Hossain, and M. Hassan, "Modeling and analysis of product line variants," in Proc. the 16th International Software Product Line Conference, vol. 2, New York, NY, USA: ACM, 2012, pp. 26-31

[17] M. Mannion, "Using first-order logic for product line model validation," in Proc. the Second International Conference on Software Product Lines, London, UK: Springer-Verlag, 2002, pp. 176-187.

[18] J. Sun, H. Zhang, and H. Wang, "Formal semantics and verification for feature modeling," in Proc. the 10th IEEE International Conference on Engineering of Complex Computer Systems, Washington, DC, USA: IEEE Computer Society, 2005, pp. 303-312.

[19] D. Benavides, P. Trinidad, and A. Ruiz-Cortés, "Automated reasoning on feature models," in Proc. the 17th international conference on Advanced Information Systems Engineering, Berlin, Heidelberg: Springer-Verlag, 2005, pp. 491-503.

[20] W. Zhang, H. Zhao, and H. Mei, "A Propositional Logic-Based Method for Verification of Feature Models," J. Davies, W. Schulte, M. Barnett, Ed., Formal Methods and Software Engineering, vol. 3308 of LNCS, Springer Berlin / Heidelberg, 2004, pp. 115-130.

[21] P. Trinidad, D. Benavides, A. Ruiz-Cortés, S. Segura, and A. Jimenez, "Fama framework," in Proc. the 2008 12th International Software Product Line Conference, Washington, DC, USA: IEEE Computer Society, 2008, pp. 359.

[22] H. H. Wang, Y. F. Li, J. Sun, H. Zhang, and J. Pan, "Verifying feature models using owl," Web Semant, vol. 5, no. 2, June 2007, pp. 117-129.

[23] M. Noorian, A. Ensan, E. Bagheri, H. Boley, and Y. Biletskiy, "Feature model debugging based on description logic reasoning," $D M S$, Knowledge Systems Institute, 2011, pp. 158-164.

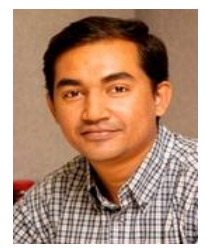

Shamim Ripon is an assistant professor in the Department of Computer Science and Engineering, East West University, Dhaka, Bangladesh where he leads Software Engineering and Formal Method Research Group. Previously, he was a Research Associate in the Department of Computing Science, University of York, UK and Research Fellow in the Department of Computing Science, University of Glasgow, UK. He also served as a Lecturer in Khulna University, Bangladesh. He is a member 
of IAENG, Senior member of IACSIT

Dr. Ripon holds a B.Sc. in Computer Science and Engineering from Khulna University, M.Sc. in Computer Science from National University of Singapore and $\mathrm{PhD}$ in Computer Science from University of Southampton, UK. His research interests focus on the Requirement Engineering, Software Product Line, Semantic Web, Natural Language Processing. His current research examines the formal representation and verification of knowledge based requirement specification.

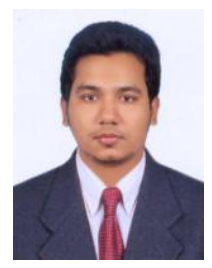

Moshiur Mahamud Piash is a B.Sc. final year student in the Department of Computer Science and Engineering, East West University, Dhaka, Bangladesh where he is involved in the Software Engineering and Formal Method Research Group.

Mr. Piash is currently working in the product line based requirement verification using semantic web. His is also interested in Web services and Intelligent Agent.

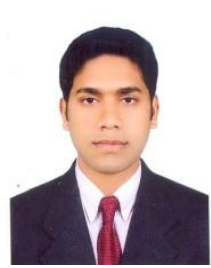

Sheikh Md. Alam Hossainis a B.Sc. final year student in the Department of Computer Science and Engineering, East West University, Dhaka, Bangladesh where he is involved in the Software Engineering and Formal Method Research Group.

Mr. Alam is currently working in the product line based requirement verification using semantic web. His research interests include Web services and Knowledge Based Software Engineering .

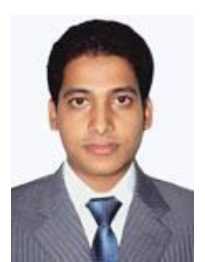

Mohammed Salah Uddin is a B.Sc. final year student in the Department of Computer Science and Engineering, East West University, Dhaka, Bangladesh where he is involved in the Software Engineering and Formal Method Research Group.

Mr. Salah Uddin is currently working towards formal verification of web services composition. He is also interested in semantic web based requirement analysis of product line requirements. 